\title{
Reentrant Pinning, Dynamic Row Reduction, and Skyrmion Accu- mulation for Driven Skyrmions in Inhomogeneous Pinning Arrays
}

\author{
C. ReichHardT ${ }^{1,2}$ and C. J. O. ReichHARDT ${ }^{1,2}$ \\ 1' Theoretical Division and Center for Nonlinear Studies, Los Alamos National Laboratory, Los Alamos, New Mexico \\ $87545, U S A$ \\ Center for Nonlinear Dynamics, Los Alamos National Laboratory, Los Alamos, New Mexico 87545, USA \\ Missing PACS ***

\begin{abstract}
We examine the dynamics of skyrmions in systems with a region of strong pinning coexisting with a pin free region, where the equilibrium state has a uniform skyrmion density. Under an applied drive, skyrmions accumulate in the pin free region along the edge of the pinned region due to the skyrmion Hall effect. As the drive increases, a series of dynamical structural transitions occur in the flowing skyrmion lattice similar to those observed in the compression dynamics of crystals. These transitions correspond to reductions in the number of flowing rows of skyrmions due to the collective motion of the skyrmions into the pinned region, and they are accompanied by a series of steps in the velocity force curves. When the number of pinning sites is sufficiently large, a drive induced pinning effect can occur when the skyrmion Hall effect forces all of the skyrmions to enter the strongly pinned region. This reentrant pinning effect becomes more pronounced for increasing intrinsic skyrmion Hall angle.
\end{abstract}

Introduction. - Skyrmions are particle like magnetic t'extures which were initially observed in neutron scattering 1 and, shortly afterwards, directly imaged with Lorentz microscopy 2. Since then, skyrmions have been identified in an increasing number of materials, including some which support skyrmions at room temperature [3]. Skyrmions have many similarities to vortex lattices in type-II superconductors, including the fact that they form a triangular lattice and can be set into motion with an applied current 3. When quenched disorder is present, there is a finite depinning threshold above which the skyrmions enter a sliding state 8 13. One aspect of skyrmions that is significantly different from superconducting vortices is that the dynamics of each skyrmion is strongly influenced by the Magnus or gyroscopic force 3, 8, 9, 11, 14, 16. In the absence of pinning, the Magnus force causes the skyrmions to move at an angle with respect to the drive called the skyrmion Hall angle, and when pinning is present, the skyrmions undergo a spiraling motion in or around the pinning sites [3. If there is no disorder, the skyrmion moves at the intrinsic skyrmion Hall angle $\theta_{s k}^{\text {int }}$ which is independent of the drive; however, when pinning is present, the skyrmion Hall angle becomes strongly drive dependent, increasing with drive from a value near zero just above depinning and eventu- ally saturating at a value close to the intrinsic skyrmion Hall angle. The drive dependent skyrmion Hall angle has been observed in particle based simulations [11,16.17, micromagnetic simulations [18 20] and experiments 20 23.

Since the skyrmions move at an angle to the drive, the skyrmion Hall effect can lead to an accumulation of skyrmions along the edge of a sample. One of the limitations in using skyrmions for devices such as race track memory is that a skyrmion does not simply travel down the race track but moves toward the track edge, where it can escape. When the Magnus force is larger, the distance the skyrmion can travel down the track is shorter [24,25], so there have been various efforts to reduce the skyrmion Hall angle [23, 25, 26]. Another approach for guiding skyrmions is to create a controlled pinning landscape that can be used to control the skyrmion motion. For example, inhomogeneous pinning or regions with strong pinning coexisting with regions of low or no pinning can be created using techniques similar to those employed for controlling the motion of vortices in type-II superconductors with nanostructured pinning arrays 27, 30.

Here we use particle based simulations to study skyrmion dynamics in a system containing a pinned region in the form of a stripe coexisting with a pin-free region, where the equilibrium state is a uniform skyrmion lattice. 

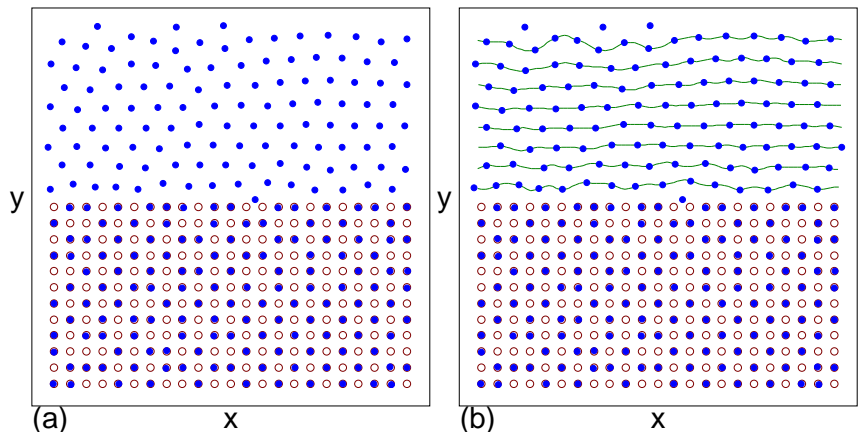

Fig. 1: Skyrmion positions (dots) and pinning sites (open circles) for a system with $\alpha_{m} / \alpha_{d}=1.25$ containing pinning in only the lower half of the sample. The drive $F_{D}$ is applied along the $x$ direction. The ratio $N_{s} / N_{p}$ of skyrmions to pinning sites in the entire sample is $1: 1$. (a) The skyrmion positions after annealing at $F_{D}=0$. (b) Skyrmion positions and trajectories (lines) at $F_{D}=0.025$ showing motion only in the unpinned region.

Under a drive applied parallel to the pinning stripe, the skyrmions in the pinned region remain immobile and the skyrmions in the unpinned region are guided parallel to the drive by the confining effect of the pinned skyrmions, giving a skyrmion Hall angle of zero for an extended range of drives. This is accompanied by the accumulation of skyrmions along the edge of the pinned region, forming a skyrmion density gradient. As the drive increases, the skyrmions in the unpinned region become increasingly compressed, resulting in sudden rearrangements of the skyrmion structure. Groups of skyrmions enter the pinned portion of the sample in order to maintain an integer number of skyrmion rows flowing in the unpinned regions, producing stick-slip jumps in the velocity-force curves along with regions of negative differential conductivity. The row reduction events in the skyrmion structure resemble those found for dynamical compression of crystals 31 and particle flow in channels $32+34$. When the drive is sufficiently large, the skyrmions can move all the way through the pinned region, giving a finite skyrmion Hall angle. At even higher drives, the skyrmion density becomes uniform again. If the number of pinning sites is large enough, it is possible to observe a drive-induced pinning effect in which, as the current increases, all the skyrmions become trapped in the pinned region. This reentrant pinning is enhanced for larger Magnus force since the skyrmions in the unpinned region are more strongly pressed against the pinned region, while in the overdamped or vortex limit, the lattice compression dynamics and reentrant pinning are lost. We map out the dynamics in a series of phase diagrams and discuses how these effects should be a general feature of skyrmion dynamics whenever the pinning is inhomogeneous.

Simulation. - We consider a two-dimensional system with periodic boundary conditions in the $x$ and $y$ - directions where only half of the sample contains $N_{p}$ pinning sites, as illustrated in Fig. 1(a). The pinning sites are placed in a square lattice with lattice constant $a$. The sample contains $N_{s}$ skyrmions, and the initial state is obtained through simulated annealing. Due to the repulsive interactions between the skyrmions, in the absence of a drive the skyrmion density is uniform, as shown in Fig. 1(a). After annealing, a drive is applied in the positive $x$-direction. We use a particle based model for the skyrmion dynamics in the presence of disorder as employed in previous works [11, 15, 17, 35]. The skyrmion dynamics is governed by the following equation of motion:

$$
\alpha_{d} \mathbf{v}_{i}+\alpha_{m} \hat{z} \times \mathbf{v}_{i}=\mathbf{F}_{i}^{s s}+\mathbf{F}^{D}
$$

where $\mathbf{v}_{i}$ is the velocity of skyrmion $i$. The repulsive skyrmion-skyrmion interaction force is $\mathbf{F}_{i}=$ $\sum_{j=1}^{N} K_{1}\left(r_{i j}\right) \hat{\mathbf{r}}_{i j}$, where $r_{i j}=\left|\mathbf{r}_{i}-\mathbf{r}_{j}\right|, r_{i j}$ is the distance between skyrmions $i$ and $j$, and the modeled Bessel function $K_{1}(r)$ falls off exponentially for large $r$. The driving force $\mathbf{F}^{D}=F^{D} \hat{\mathbf{x}}$ is applied uniformly to all particles in the direction parallel to the stripe of pinning. We measure the skyrmion velocity both parallel, $\left\langle V_{||}\right\rangle=N_{s}^{-1} \sum_{i}^{N_{s}} \mathbf{v}_{i} \cdot \hat{\mathbf{x}}$, and perpendicular, $\left\langle V_{\perp}\right\rangle=N_{s}^{-1} \sum_{i}^{N_{s}} \mathbf{v}_{i} \cdot \hat{\mathbf{y}}$, to the driving force. We increase the drive in increments of $\Delta F_{D}=$ 0.0002 and average over 75000 time steps at each drive to ensure that the dynamics is in a steady state. The damping term $\alpha_{d}$ aligns the skyrmion velocity in the direction of the net applied force, while $\alpha_{m}$ is the coefficient of the Magnus term which creates a velocity component perpendicular to the net applied force. For a finite Magnus term, in the of absence pinning the skyrmions move at an angle with respect to the driving force of $\theta_{s k}^{\text {int }}=\arctan \left(\alpha_{m} / \alpha_{d}\right)$. In Fig. 11 the drive is applied in the positive $x$-direction, and the resulting skyrmion velocity is in the positive $x$ and negative $y$ direction. The pinning is modeled as parabolic traps of radius $r_{p}=0.25$ with a maximum strength of $F_{p}$. The density of the system is given by $N_{s} / N_{p}$, the ratio of the number of skyrmions in the entire sample to the total number of pinning sites. In Fig. 1 $N_{s} / N_{p}=1.0$. To perform the simulated annealing, we start in a higher temperature liquid state and gradually cool the sample to $T=0$. Generally this produces a state with a uniform skyrmion density, as illustrated in Fig. 1(a) for $F_{D}=0.0$. Under an applied drive, the skyrmions in the unpinned region depin first, as shown in Fig. 1(b) at $F_{D}=0.025$, where the skyrmion motion is confined along the $x$ direction.

Shearing Dynamics for Parallel Driving. - In Fig. 2 we plot $\left\langle V_{\|}\right\rangle$and $\left\langle V_{\perp}\right\rangle$ versus $F_{D}$ for the the system

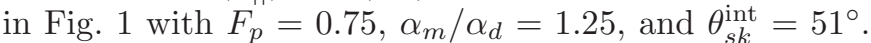
For $0<F_{D}<0.47$, the motion is strictly along the direction of drive, with $\left\langle V_{\perp}\right\rangle=0$, and a series of sharp velocity drops appear in $\left\langle V_{\|}\right\rangle$. This is region $\mathrm{I}$, a shear flow phase, in which only the skyrmions in the unpinned portion of the sample are moving. For $0.45 \leq F_{D}<0.72$, these drops disappear and both $\left\langle V_{\|}\right\rangle$and $\left\langle V_{\perp}\right\rangle$ increase 


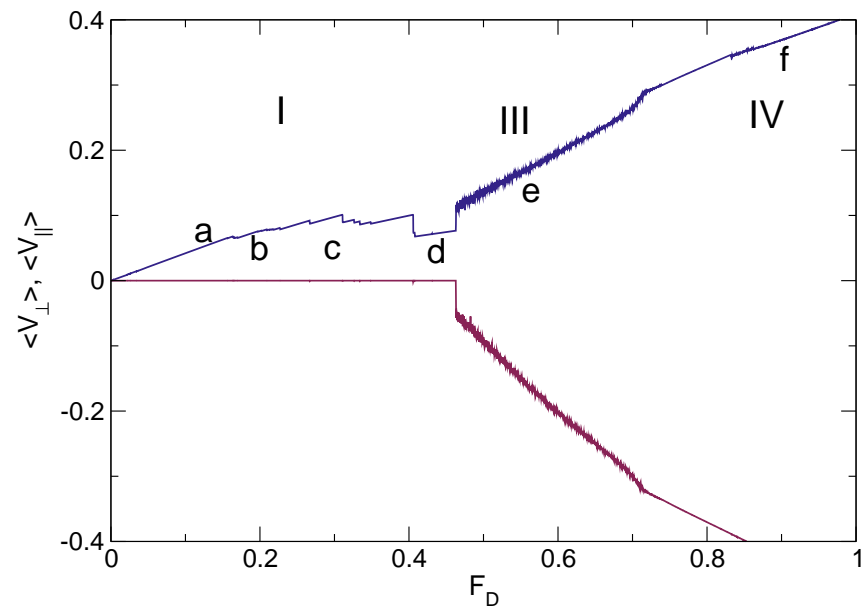

Fig. 2: The average skyrmion velocity parallel, $\left\langle V_{\|}\right\rangle$(blue), and perpendicular, $\left\langle V_{\perp}\right\rangle$ (red), to the drive vs $F_{D}$ for the system in Fig. 1 1 with $F_{p}=0.75$. Region $\mathrm{I}$ is the shear flow where the skyrmions in the pin-free region move in the direction of drive, as illustrated in Fig. 3(a,b,c,d). Region III is the disordered plastic flow where motion occurs in both directions, as shown in Fig. $3(\mathrm{e})$, and in region IV, all the skyrmions are moving in a lattice structure as in Fig. 3 (f).

with increasing $F_{D}$. Here the sample has entered region III, a disordered plastic flow state, where motion occurs both parallel and perpendicular to the drive, but some skyrmions remain pinned and the overall skyrmion structure is disordered. For $F_{D}>0.72$, there is a change in the slope and the velocity in both directions increases linearly with drive. This is region IV, where all the skyrmions form a uniform lattice that moves in a direction that is close to the intrinsic skyrmion Hall angle.

In Fig. 1(b) at $F_{D}=0.025$, the skyrmions in the unpinned region form a moving uniform triangular lattice. As $F_{D}$ increases, the Magnus term pushes these skyrmions in the negative $y$ direction, causing the top row of pinning sites to fill with skyrmions and producing a density gradient of skyrmions in the unpinned area, as shown in Fig. 3(a) for $F_{D}=0.125$. Here there are seven rows of moving skyrmions in the unpinned region. As $F_{D}$ is increased further, the skyrmions become more compressed and the number of moving rows drops, as shown in Fig. 3(b) at $F_{D}=0.225$ where there are six rows of moving skyrmions. The sharp drops in $\left\langle V_{\|}\right\rangle$in Fig. 2 correspond to drives at which the compression of the skyrmion structure generates row reduction and partial row reduction events. At $F_{D}=0.275$ in Fig. 3(c), there are four rows of moving skyrmions, as highlighted by the plot of the skyrmion trajectories. After a series of additional small jumps in $\left\langle V_{\|}\right\rangle$, there are three rows of moving skyrmions for $0.35<F_{D}<0.4$, and for $0.4<F_{D}<0.465$, there are only two rows of moving skyrmions, as shown in Fig. 3(d) at $F_{D}=0.425$. Once the drive is large enough, the skyrmions in the pinned region begin to flow, and the systems enters a disordered plastic flow state in which the
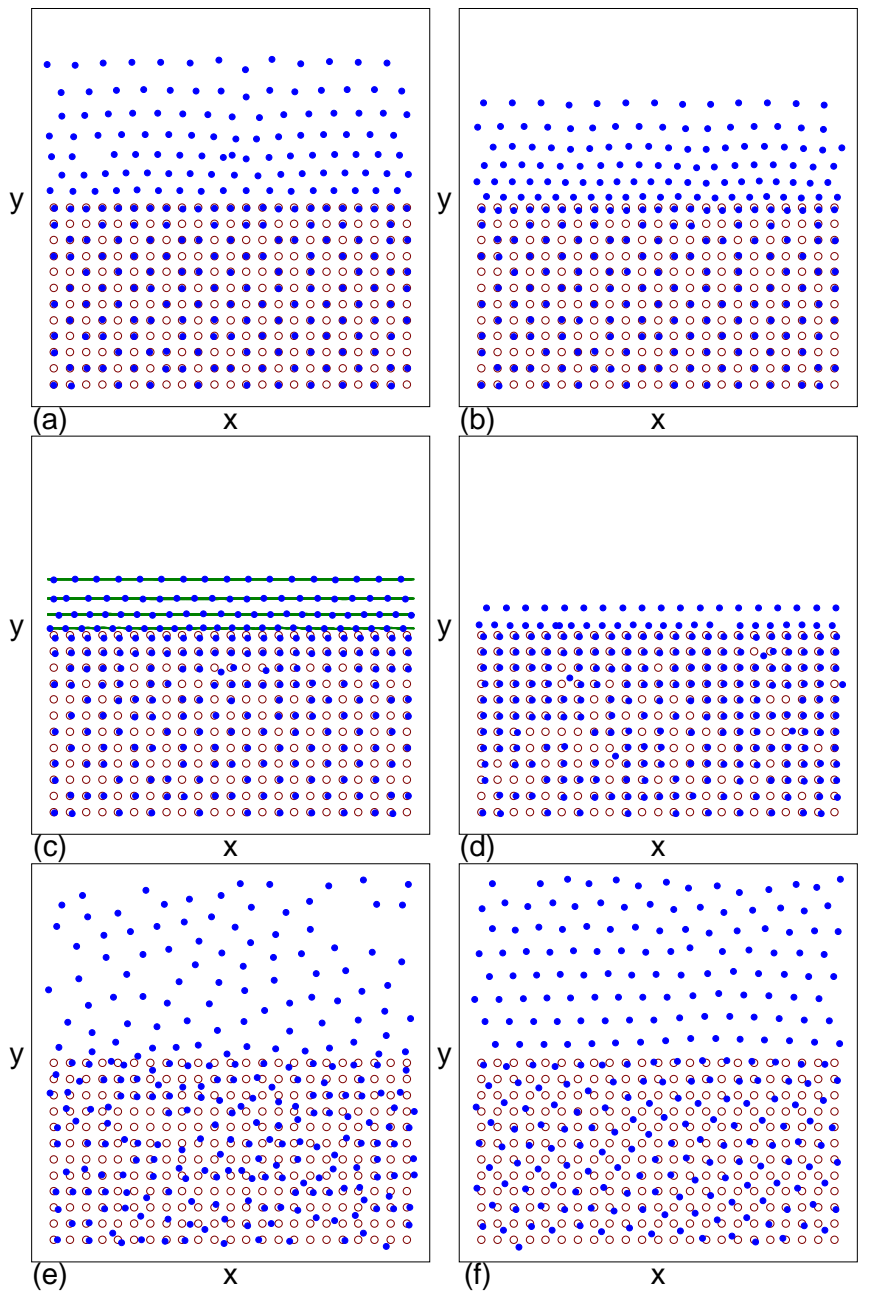

Fig. 3: Skyrmion positions (dots) and pinning sites (open circles) for the system in Fig. 2 at the points marked a through f. (a) At $F_{D}=0.125$, the top row of pinning sites next to the unpinned region is filled and a skyrmion density gradient starts to form in the unpinned region. (b) At $F_{D}=0.225$, there are six rows of moving skyrmions. (c) At $F_{D}=0.325$, where the skyrmion trajectories (lines) are highlighted, there are four rows of moving skyrmions. (d) At $F_{D}=0.425$, there are only two rows of moving skyrmions. (e) $F_{D}=0.6$ in the disordered plastic flow region III. (f) $F_{D}=0.9$ in region IV or the moving lattice state.

skyrmions can move both parallel and perpendicular to the drive. Figure 3(e) illustrates the skyrmion structure in region III at $F_{D}=0.6$. At the highest drives, the system enters a flowing lattice phase, as shown in Fig. 3(f) at $F_{D}=0.9$. The moving lattice state is similar to that observed at higher drives in simulations with uniform pinning where the skyrmions dynamically reorder into a crystal structure [11, 13].

The drops in the velocity-force curves indicate that the system exhibits negative differential conductivity with $d\left\langle V_{\| \mid}\right\rangle / d F_{D}<0$. Similar effects have been observed for vortices in periodic pinning arrays at transitions from disor- 

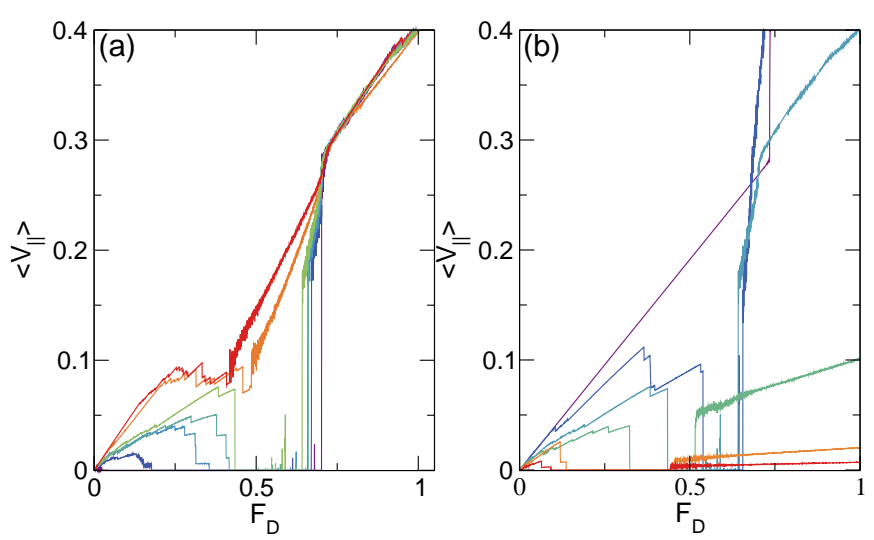

Fig. 4: (a) $\left\langle V_{\|}\right\rangle$vs $F_{D}$ for the system in Fig. 2 at fixed $\alpha_{m} / \alpha_{d}=1.25$ and $F_{p}=0.75$ for $N_{s} / N_{p}=1.1$ (red), 0.375 (orange), 0.3125 (light green), 0.25 (blue green), 0.1875 (light blue), 0.125 (dark blue), and 0.0625 (purple). Here there is a reentrant pinning region II for $N_{s} / N_{p}<0.5$. (b) $\left\langle V_{\|}\right\rangle$vs $F_{D}$ for the same system at $N_{s} / N_{v}=0.3125$ and $\alpha_{m} / \alpha_{d}=0.0$ (purple), 0.75 (dark blue), 1.25 (light blue), 3.0 (blue green), 9.0 (orange), and 18.0 (red). Here region II is lost for $\alpha_{m} / \alpha_{d}=0$ but becomes extended for increasing $\alpha_{m} / \alpha_{d}$.

dered two-dimensional flow to effectively one-dimensional flow, where the number of moving vortices drops as the drive increases [36, 37]. In the vortex system, there is only one velocity drop, while in the skyrmion system there are a series of drops. The sudden changes in the skyrmion lattice structure during compression are similar to what is found in the compression of charged particles by time dependent potentials with increasing confining strength, where the compressed lattice undergoes a combination of slow elastic deformations interspersed with sudden drops due to plastic rearrangements, and where the highest stability occurs when an integer number of rows of particles can form a triangular lattice within the confined region 31. In the skyrmion case, the compression is produced by the increase in the Magnus force with increasing drive that pushes the moving skyrmions against the pinned region, with the pinned skyrmions acting as a barrier. For varied filling ratios $N_{s} / N_{p}$, we observe similar features in the velocity force curves, and when the number of skyrmions is much smaller than the number of pinning sites, we find a drive-induced pinning phenomenon in which all the skyrmions leave the unpinned region and become trapped in the pinned region.

In Fig. $4(\mathrm{a})$ we plot $\left\langle V_{\|}\right\rangle$versus $F_{D}$ for the same system as in Fig. 2 at varied skyrmion density of $N_{s} / N_{p}=$ $1.1,0.375,0.3125,0.25,0.1875,0.125$, and 0.0625 . For $N_{s} / N_{p}=1.1$ we find the same phases I, III, and IV as in Fig. 2, but for $N_{s} / N_{p}<0.5$ we observe a new reentrant phase, as shown in the $N_{s} / N_{p}=0.3125$ curve which has a transition from phase I flow to a state with $\left\langle V_{\|}\right\rangle=0$ over the range $0.5125<F_{D}<0.575$. In this same interval, $\left\langle V_{\perp}\right\rangle=0$ (not shown). We label this reentrant pinning effect region II, and as $N_{s} / N_{p}$ decreases, the onset of phase
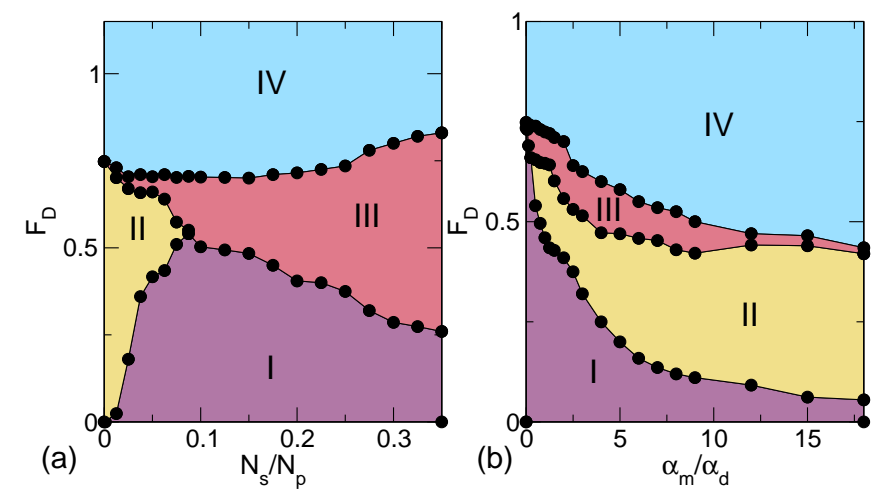

Fig. 5: (a) Dynamic phase diagram for the system in Fig. 4(a) as a function of $F_{D}$ vs $N_{s} / N_{p}$ at fixed $\alpha_{m} / \alpha_{d}=1.25$. I: shear flow (light purple); II: reentrant pinning (yellow); III: disordered plastic flow (pink); IV: uniform moving crystal (blue). (b) Dynamic phase diagram for the same system as a function of $F_{D}$ vs $\alpha_{m} / \alpha_{d}$ at fixed $N_{s} / N_{p}=0.3125$.

\section{II shifts to lower values of $F_{D}$.}

Since the reentrant phase arises when the Magnus force pushes the skyrmions into the pinned region, we also examine the effect of changing $\alpha_{m}$ while fixing $\alpha_{d}=1.0$. In Fig. 4(b) we plot $\left\langle V_{\|}\right\rangle$versus $F_{D}$ for a system with $N_{s} / N_{p}=0.3125$ at $\alpha_{m} / \alpha_{d}=0.0,0.75,1.25,3.0,9.0$, and 18.0. In the overdamped case of $\alpha_{m} / \alpha_{d}=0.0$, the drop in $\left\langle V_{\| l}\right\rangle$ disappears and the velocity increases monotonically with increasing $F_{D}$. For $\alpha_{m} / \alpha_{d}<0.35$ there is no reentrant pinning phase since the skyrmion Hall angle is too small; however, for $\alpha_{m}>0.35$, phase II appears and becomes wider with increasing $\alpha_{m} / \alpha_{d}$ as the skyrmion move more rapidly into the pinned region. The onsets of phases III and IV also shift to lower $F_{D}$ with increasing $\alpha_{m}$.

In Fig. 5(a) we plot the dynamic phase diagram as a function of $F_{D}$ versus the filling fraction $N_{s} / N_{p}$ for the system in Fig. 4(a) at $\alpha_{m} / \alpha_{d}=1.25$. Here, phase II appears only for $N_{s} / N_{p}<0.1$, while the width of phase I decreases with increasing $N_{s} / N_{p}$. The III-IV transition depends only weakly on the filling, and the system can dynamically reorder when $F_{D} / F_{p}>1.0$. For the lowest filling of $N_{s} / N_{p}<0.05$, region IV is a uniform moving liquid rather than a moving lattice since the skyrmions are far enough apart that the skyrmion-skyrmion interactions are relatively weak. Figure 5(b) shows the dynamic phase diagram as a function of $F_{D}$ versus $\alpha_{m} / \alpha_{d}$ in the same system at $N_{s} / N_{p}=0.3125$, highlighting the fact that the width of phase II increases with increasing $\alpha_{m}$.

Phase II can occur for fillings $N_{s} / N_{p}>0.5$ depending on the strength of the pinning. In Fig. 6(a) we plot $\left\langle V_{\|}\right\rangle$versus $F_{D}$ for a system with $\alpha_{m} / \alpha_{d}=1.25$ at $N_{s} / N_{p}=1.0$ with $F_{p}=0.25,2.0$, and 2.25 , showing that as $F_{p}$ increases, the extent of region I grows, and that when $F_{p}>2.0$, the reentrant pinning phase II can occur. In Fig. 6(b) we illustrate the dynamic phase digram as a function of $F_{D}$ versus $F_{p}$ for the system in Fig. 6(a), high- 

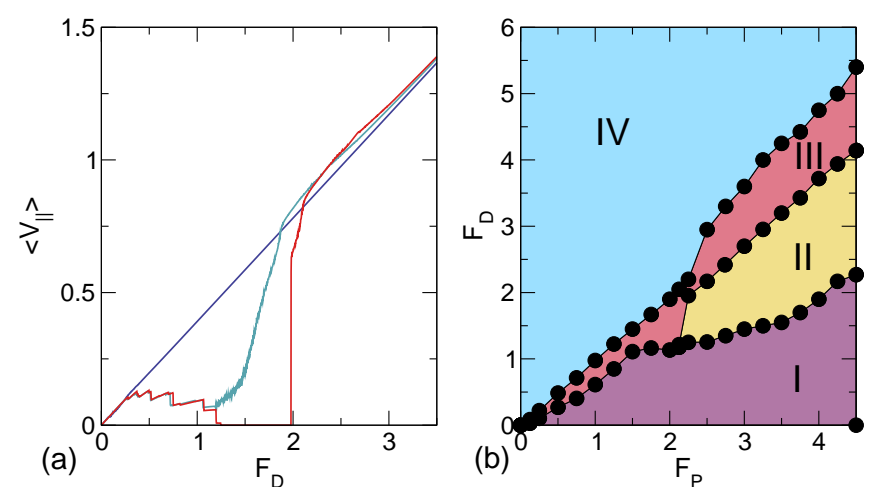

Fig. 6: (a) $\left\langle V_{\|}\right\rangle$vs $F_{D}$ for a system with $\alpha_{m} / \alpha_{d}=1.25$ and $N_{s} / N_{p}=1.0$ at $F_{p}=0.25$ (dark blue), 2.0 (blue green), and 2.25 (red), showing that phase II can emerge for increased pinning strength. (b) Dynamic phase diagram as a function of $F_{D}$ vs $F_{p}$ for the system in (a) showing that phase II occurs when $F_{p}>2.0$.

lighting phases I-IV. Reentrant pinning can occur when $F_{p}>2.0$. At $N_{s} / N_{p}>1.0$, there is always some skyrmion motion in the sample; however, there are still intervals of $F_{D}$ in which $\left\langle V_{\|}\right\rangle$is small. In general, for a system with either strong pinning or large maximum forces on the skyrmions, whenever the pinning is inhomogeneous we would expect to find both the shear flow phase I and the reentrant pinning phase, along with an accumulation of skyrmions along the edges of the pinned region. Recent experiments have demonstrated drive-induced skyrmion accumulation and skyrmion density gradients along the sample edges, and these effects were attributed to the Magnus force 38. There has also been previous work on drive-induced pinning effects for single skyrmions interacting with magnetic pinning sites [39]; however, this is a different effect than what we describe since in Ref. 39, the pinning sites produce a repulsive potential barrier that the skyrmion must overcome at finite drive in order to be captured.

In summary, we investigate skyrmion dynamics in a system with spatially inhomogeneous pinning, where a square pinning array occupies only half of the sample in a stripe aligned with the driving direction. In the equilibrium state, the skyrmion density is uniform, and under an applied drive, skyrmion motion initially occurs only in the unpinned region and is in the same direction as the driving force. As the drive increases, the skyrmions in the unpinned region begin to form a density gradient due to the Magnus force, with an accumulation of skyrmions appearing at the edge of the pinned region. A series of drops in the velocity-force curves occur when sudden rearrangements of the moving skyrmions occur as the compression of the skyrmion lattice increases with increasing drive. The jumps are correlated with partial row reduction events of the moving skyrmions as groups of skyrmions become trapped in the pinned region. At higher drives, there is a transition to a disordered plastic flow state fol- lowed by a transition to a moving lattice state, in both of which the skyrmions move both parallel and perpendicular to the driving force due to the Magnus term. When the number of pinning sites is sufficiently high, a reentrant pinning phenomenon appears where there is a finite drive at which all the skyrmions become trapped in the pinned region. The reentrant pinning regime increases in extent for increasing intrinsic skyrmion Hall angle since the skyrmions can move more rapidly into the pinning sites. In the overdamped limit, the reentrant pinning and dynamical skyrmion lattice compression are lost. Our results indicate that inhomogeneous pinning can be used to guide skyrmions, and that the skyrmion gradient and accumulation should be general features of skyrmion systems with inhomogeneous disorder.

$$
* * *
$$

This work was supported by the US Department of Energy through the Los Alamos National Laboratory. Los Alamos National Laboratory is operated by Triad National Security, LLC, for the National Nuclear Security Administration of the U. S. Department of Energy (Contract No. 892333218NCA000001).

\section{REFERENCES}

[1] Mühlbauer S., Binz B., Jonietz F., Pfleiderer C., Rosch A., Neubauer A., Georgit R. and Böni P., Science, 323 (2009) 915.

[2] Yu X. Z., Onose Y., Kanazawa N., Park J. H., Han J. H., Matsui Y., Nagaosa N. and Tokura Y., Nature (London), 465 (2010) 901.

[3] Nagaosa N. and Tokura Y., Nature Nanotechnol., 8 (2013) 899.

[4] Jiang W., Upadhyaya P., Zhang W., Yu G., Jungfleisch M. B., Fradin F. Y., Pearson J. E., Tserkovnyak Y., Wang K. L., Heinonen O., te Velthuis S. G. E. and Hoffmann A., Science, 349 (2015) 283.

[5] Woo S., Litzius K., Krüger B., Im M.-Y., Caretta L., Richter K., Mann M., Krone A., Reeve R. M., Weigand M., Agrawal P., Lemesh I., Mawass M.A., Fischer P., Kläui M. and Beach G. S. D., Nature Mater., 15 (2016) 501.

[6] Boulle O., Vogel J., Yang H., Pizzini S., de Souza Chaves D., Locatelli A., Mentes T. O., Sala A., Buda-Prejbeanu L. D., Klein O., Belmeguenai M., Roussigné Y., Stashkevich A., Chérif S. M., Aballe L., Chshiev M. F. M., Auffret S., Miron I. M. and Gaudin G., Nature Nanotechnol., 11 (2016) 449.

[7] Soumyanarayanan A., Raju M., Gonzalez-Oyarce A. L., Tan A. K. C., Im M.-Y., Petrovic A. P., Ho P., Khoo K. H., Tran M., Gan C. K., Ernult F. and Panagopoulos C., Nature Mater., 16 (2017) 898.

[8] Schulz T., Ritz R., Bauer A., Halder M., Wagner M., Franz C., Pfleiderer C., Everschor K., Garst M. and Rosch A., Nature Phys., 8 (2012) 301. 
[9] Iwasaki J., Mochizuki M. and Nagaosa N., Nat. Commun., 4 (2013) 1463.

[10] Liang D., DeGrave J. P., Stolt M. J., Tokura Y. and Jin S., Nature Commun., 6 (2015) 8217.

[11] Reichhardt C., Ray D. and Reichhardt C. J. O., Phys. Rev. Lett., 114 (2015) 217202.

[12] Tolley R., Montoya S. and Fullerton E., Phys. Rev. Mater., 2 (2018) 044404.

[13] Koshibae W. and Nagaosa N., Sci. Rep., 8 (2018) 6328.

[14] Kim B. S., J. Phys.: Condens. Matt., 31 (2019) 383001.

[15] Brown B. L., Täuber U. C. and Pleimling M., Phys. Rev. B, 97 (2018) 020405(R).

[16] Reichiardt C. and Reichhardt C. J. O., New J. Phys., 18 (2016) 095005.

[17] Díaz S. A., Reichhardt C. J. O., Arovas D. P., Saxena A. and Reichhardt C., Phys. Rev. B, 96 (2017) 085106.

[18] Legrand W., Maccariello D., Reyren N., Garcia K., Moutafis C., Moreau-Luchaire C., Collin S., Bouzehouane K., Cros V. and Fert A., Nano Lett., 17 (2017) 2703.

[19] Kim J.-V. and Yoo M.-W., Appl. Phys. Lett., 110 (2017) 132404.

[20] Juge R., Je S.-G., De Souza Chaves D., BudaPrejbeanu L. D., Peña-Garcia J., Nath J., Miron I. M., Rana K. G., Aballe L., Foerster M., Genuzio F., Mentes T. O., Locatelli A., Maccherozzi F., Dhesi S. S., Belmeguenai M., Roussigné Y., Auffret S., Pizzini S., Gaudin G., Vogel J. and Boulle O., arXiv e-prints, (2019) arXiv:1904.08275.

[21] Jiang W., Zhang X., Yu G., Zhang W., Wang X., Jungfleisch M. B., Pearson J. E., Cheng X., Heinonen O., Wang K. L., Zhou Y., Hoffmann A. and te Velthuis S. G. E., Nature Phys., 13 (2017) 162.

[22] Litzius K., Lemesh I., Krüger B., Bassirian P., Caretta L., Richter K., Büttner F., Sato K., Tretiakov O. A., Förster J., Reeve R. M., Weigand M., Bykova I., Stoll H., Schütz G., Beach G. S. D. and KLÄUi M., Nature Phys., 13 (2017) 170.

[23] Woo S., Song K. M., Zhang X. C., Zhou Y., Ezawa M., Liu X. X., Finizio S., Raabe J., Lee N. J., Kim S. I., PARK S. Y., Kim Y., Kim J. Y., LeE D., LeE O., Choi J. W., Min B. C., Koo H. C. and Chang J., Nature Commun., 9 (2018) 959.

[24] Iwasaki J., Mochizuki M. and Nagaosa N., Nature Nanotechnol., 8 (2013) 742.

[25] Everschor-Sitte K., Masell J., Reeve R. M. and KläUi M., J. Appl. Phys., 124 (2018) 240901.

[26] Hirata Y., Kim D.-H., Kim S. K., Lee D.-K., Oh S.H., Kim D.-Y., Nishimura T., Okuno T., Futakawa Y., Yoshikawa H., Tsukamoto A., Tserkovnyak Y., Shiota Y., Moriyama T., Choe S.-B., Lee K.-J. and Ono T., Nature Nanotechnol., 14 (2019) 232.

[27] Harada K., Kamimura O., Kasai H., Matsuda T., Tonomura A. and Moshchalkov V. V., Science, 274 (1996) 1167.

[28] Martín J. I., Vélez M., Nogués J. and Schuller I. K., Phys. Rev. Lett., 79 (1997) 1929.

[29] Welp U., Xiao Z. L., Novosad V. and VlaskoVlasov V. K., Phys. Rev. B, 71 (2005) 014505.

[30] Trastoy J., Malnou M., Ulysse C., Bernard R., Bergeal N., Faini G., Lesueur J., Briatico J. and
Villegas J. E., Nature Nanotechnol., 9 (2014) 710.

[31] McDermott D., Reichhardt C. J. O. and Reichhardt C., Phys. Rev. E, 93 (2016) 062607.

[32] Konubo N., Besseling R. and Kes P. H., Phys. Rev. $B, 69$ (2004) 064504.

[33] Besseling R., Niggebrugge R. and Kes P. H., Phys. Rev. Lett., 82 (1999) 3144.

[34] Besseling R., Kes P. H., Dröse T. and Vinokur V. M., New J. Phys., 7 (2005) 71.

[35] Lin S.-Z., Reichhardt C., Batista C. D. and Saxena A., Phys. Rev. B, 87 (2013) 214419.

[36] Reichhardt C., Olson C. J. and Nori F., Phys. Rev. Lett., 78 (1997) 2648.

[37] Gutierrez J., Silhanek A. V., Van de Vondel J., Gillijns W. and Moshchalkov V. V., Phys. Rev. B, 80 (2009) 140514.

[38] Sugimoto S., Kasai S., Anokhin E., Takahashi Y. and Tokura Y., Appl. Phys. Express, 12 (2019) 073002.

[39] Müller J. and Rosch A., Phys. Rev. B, 91 (2015) 054410 . 